\title{
Maintenance prédictive de l'outil de coupe en usinage
}

\author{
Rachid Noureddine ${ }^{1}$, Ali Benamar ${ }^{2}$ et Farid Noureddine ${ }^{3, a}$ \\ 1 Université d'Oran, BP 1524 El-M'Naouer, 31000 Oran, Algérie \\ 2 ENSET Oran, BP 1523 El-M'Naouer, 31000 Oran, Algérie \\ 3 ENI Tarbes, 47 avenue d'Azereix, 65016 Tarbes Cedex, France
}

Reçu le 29 mai 2007, accepté le 14 février 2008

\begin{abstract}
Résumé - Le travail que nous présentons dans cet article a pour but de montrer les avantages liés à la mise en place d'une maintenance prédictive, relative aux outils de coupe pour les usinages à enlèvement de matière. Nous décrivons la méthode de détection des fautes basée sur l'utilisation du modèle analytique de la rugosité des pièces usinées. Ce modèle est établi sous des conditions de coupe données, calculées en fonction de la rugosité maximale souhaitée. Deux types de fautes, d'une part l'usure prématurée de l'outil de coupe et d'autre part le bris de l'outil sont injectées lors de l'usinage des pièces et permettent ainsi de valider la méthode de détection proposée. Le principe de cette méthode est de comparer la rugosité mesurée à la rugosité calculée à partir du modèle analytique. En l'absence de faute, les deux valeurs traduisant ces rugosités sont identiques, tandis que l'occurrence d'une faute se traduit par une différence entre ces deux signaux, cette différence étant appelée résidu. Une maintenance systématique est également proposée pour pallier à l'usure normale de l'outil. La durée de vie de l'outil de coupe est calculée en utilisant le modèle de la rugosité couplé à la rugosité maximale spécifiée.
\end{abstract}

Mots clés : Maintenance prédictive / usinage / rugosité / détection des fautes

\begin{abstract}
Predictive maintenance in the cutting tool in machining. The purpose of this work is to show the advantages linked to the implementation of predictive maintenance, especially the maintenance in cutting tools in the process of removal of matter. The fault detection method is based on the use of the roughness model of the work pieces. This model is established on cutting conditions according to the desired maximum roughness. Two types of faults are introduced in the process to validate the proposed detection method. These faults concern on the one hand the premature wear of the cutting tool and on the other hand the breaking of the tool. The principle of the fault detection method is to compare the measured roughness with the estimated roughness thanks to the analytical model. When there is no fault these two values of the roughness are similar whereas the occurence of a fault induces a variation between these two signals. This difference is called residual. A systematic maintenance is also proposed to take in charge the normal wear of the tool. The duration of the tool life is calculated using the calculated roughness model linked with the specified maximum roughness.
\end{abstract}

Key words: Predictive maintenance / machining / roughness / fault detection

\section{Introduction}

Les stratégies de maintenance dans l'industrie manufacturière reposent principalement sur des actions de maintenance préventive systématique, dont le but est d'assurer la disponibilité des systèmes. Les ordres de maintenance sont lancés à partir de valeurs prédéterminées de durée de fonctionnement, valeurs

\footnotetext{
${ }^{a}$ Auteur pour correspondance :

farid.noureddine@enit.fr
}

généralement obtenues à partir de valeurs statistiques moyennes. Toutes les déviations recensées, inhérentes à l'utilisation du système pendant une durée donnée, sont corrigées lors de l'opération de maintenance.

Précisons d'ores et déjà la terminologie que nous adoptons au cours de nos travaux : un système technologique peut être sujet à des usures, des déviations, des défauts, des perturbations, des dysfonctionnements, des pannes, des erreurs, des défaillances, voire des fautes. Nous utiliserons dans ce travail la terminologie définie dans la communauté scientifique 


\section{Nomenclature}

\begin{tabular}{|lll|}
\hline$a$ & avance de coupe & {$\left[\mathrm{mm} \cdot \mathrm{tr}^{-1}\right]$} \\
$c$ & constante dépendante des conditions de coupe & \\
$C 38$ & acier de traitement thermique $(X C 38 f)$ & \\
$f_{1}$ & faute d'usure prématurée de l'outil de coupe & \\
$f_{2}$ & faute de bris du bec de l'outil de coupe & \\
$p$ & profondeur de passe & {$[\mathrm{mm}]$} \\
$P 20$ & nuance de carbure de l'outil & \\
$R(t)$ & rugosité & {$[\mu \mathrm{m}]$} \\
$R_{\mathrm{g}}$ & rugosité théorique de génération & {$[\mu \mathrm{m}]$} \\
$R_{\mathrm{a}}(t)$ & rugosité moyenne arithmétique & {$[\mu \mathrm{m}]$} \\
$R_{\mathrm{M}}$ & rugosité maximale imposée & {$[\mu \mathrm{m}]$} \\
$r(t)$ & résidu & {$[\mu \mathrm{m}]$} \\
$r_{\epsilon}$ & rayon d'arrondi du bec de l'outil & {$[\mathrm{mm}]$} \\
$s$ & variable de répartition de la loi normale & \\
$S_{\mathrm{H}}$ & seuil haut & {$[\mu \mathrm{m}]$} \\
$S_{\mathrm{B}}$ & seuil bas & {$[\mu \mathrm{m}]$} \\
$t$ & temps & {$[\mathrm{s}]$} \\
$V_{\mathrm{c}}$ & vitesse de coupe & {$[\mathrm{m} . \mathrm{min}$} \\
$\tau$ & valeur du temps d'un cycle d'usinage & {$[\mathrm{s}]$} \\
$\tau_{\mathrm{c}}$ & valeur du temps où la rugosité est variable & {$[\mathrm{s}]$} \\
$\tau_{\mathrm{g}}$ & valeur du temps où la rugosité est constante & {$[\mathrm{s}]$} \\
$\alpha$ & angle de dépouille de l'outil de coupe & {$\left[{ }^{\circ}\right]$} \\
$\gamma$ & angle d'attaque de l'outil de coupe & {$\left[{ }^{\circ}\right]$} \\
$\mu$ & moyenne arithmétique statistique & \\
$\sigma$ & écart type & \\
$m$ & exposant dépendant des conditions de coupe & \\
\hline & & \\
\hline
\end{tabular}

dont le domaine d'activité est la sûreté de fonctionnement des systèmes informatiques où les notions de faute, erreur et défaillance sont précisément explicitées [1].

La maintenance préventive systématique, par l'utilisation a priori d'une valeur prédéterminée de la durée de fonctionnement d'un système, peut mener soit à des interventions trop précoces donc inutiles, soit trop tardives donc préjudiciables au bon fonctionnement du système. Il est couramment admis, dans le cas de systèmes manufacturiers, que ce type de maintenance s'avère performant pour corriger les fautes d'origine systématique, dont la principale est l'usure.

Les travaux liés à la tolérance aux fautes permettent d'amener une dimension supplémentaire à la fonction maintenance. La tolérance aux fautes a pour objectif de doter le système de capacités permettant de détecter et localiser les fautes et de permettre une continuité du service par une accommodation de ces fautes, ceci devant être réalisé de façon automatique et si possible en temps réel. Le développement d'algorithmes de détection des fautes est une préoccupation constante de nombre de chercheurs et repose sur différents principes. Une des principales méthodes s'appuie sur l'utilisation du modèle analytique du processus considéré, [2] et [3].

L'accommodation des fautes est généralement obtenue par l'application de techniques de redondances matérielle et/ou logicielle suivant le système considéré, [4].

Cet apport de la communauté des automaticiens est maintenant directement exploitable dans l'implémentation de techniques de maintenance prédictive, dont le point commun avec la tolérance aux fautes est la mise en place d'étages de détection de fautes, complémentés par une prédiction de l'instant d'intervention de la logistique maintenance.

L'avantage de la maintenance prédictive par rapport à la maintenance systématique est double. Elle peut permettre de rationaliser les interventions de maintenance par rapport aux fautes systématiques (usure) et amène une dimension supplémentaire de tolérance par rapport à des fautes telles que le bris du bec de l'outil ou l'usure prématurée de ce dernier.

Après l'introduction, nous rappelons dans la seconde section les points essentiels caractérisant la maintenance prédictive, rappel qui sera suivi d'un état de l'art des travaux relatifs à ce type de maintenance.

La troisième section présente le contexte de notre étude et la stratégie de maintenance suivie. La section suivante est consacrée à la modélisation de la rugosité des pièces, cette dernière constituant l'indicateur sur lequel est basée la détection des fautes. Une étude de cas est ensuite développée et les paramètres de coupe retenus sont listés. La sixième section traite de l'usure systématique de l'outil et permet de présenter 
la maintenance systématique. La dernière section est consacrée à la maintenance prédictive de l'outil de coupe.

\section{Maintenance prédictive - État de l'art}

Parmi les différentes possibilités d'organisation et d'optimisation de la fonction maintenance dans son ensemble, la maintenance prédictive se révèle être la plus prometteuse, à la condition expresse de satisfaire aux impératifs liés aux différents étages qui la constituent.

La principale spécificité de la maintenance prédictive réside dans la prédiction du moment d'intervention en vue de pallier à la faute survenue, [5]. Le calcul de cet instant est réalisé à partir de l'analyse d'une tendance d'évolution d'un ou de plusieurs paramètres choisis pour leur pertinence. En aval de cet étage est implanté un étage de prise de décision, dont le rôle est d'arbitrer entre les différents acteurs de l'entreprise pour engager ou retarder une intervention. En amont, par contre, est présent l'étage de détectionlocalisation de la faute, qui constitue souvent le verrou scientifique d'une procédure d'implantation d'une maintenance prédictive. Trois étapes sont donc à mener dans la réalisation d'une maintenance prédictive, à savoir :

1. détection-localisation de la faute,

2. prédiction du moment d'intervention,

3. prise de décision d'intervention.

Dans le cadre du travail que nous présentons, nous focalisons essentiellement sur le point 1. Des éléments du point 2 sont également mentionnés tandis que le point 3 nécessitera un développement à part entière. Ces 3 points sont, par contre, abordés dans [6] où une plate forme de supervision est implantée sur un générateur hydroélectrique. Les éléments identifiés comme sensibles et devant faire l'objet d'une surveillance sont les servomécanismes électrohydrauliques.

Dans le cadre de la maintenance d'un parc éolien, [7] présente un plan d'application de maintenance prédictive où le paramètre surveillé est la température de différentes parties des turbines du système.

Dans les deux publications pré-citées, l'étage de détection-diagnostic des fautes est implémenté grâce à un réseau de neurones disposé en ligne. Cette approche de détection-diagnostic appartient à la classe des méthodes basées sur l'intelligence artificielle que l'on retrouve également dans [8] et [9]. Les réseaux de neurones sont également utilisés dans [10] et [11] pour élaborer l'étage de détection où le paramètre pertinent à surveiller est le courant consommé par les machines concernées par le programme de maintenance.

La détection des fautes peut aussi être menée en s'inspirant des techniques de traitement du signal, [12] et [13]. De ce fait, dans le cas des machines tournantes, l'indicateur à surveiller est souvent corrélé aux vibrations engendrées par la machine. Les techniques d'analyse spectrale sont souvent mises en œuvre pour faire apparaître les vibrations excessives et les fautes corrélées à ces vibrations.

La dernière méthode sur laquelle peut s'appuyer l'étage de détection des fautes est celle utilisant le modèle analytique du système. Cette voie est la voie privilégiée par les automaticiens, vu que ce modèle est établi lors de la phase de contrôle du système. [14] a utilisé ce principe de détection pour la conception d'un robot industriel tolérant aux fautes. Le paramètre surveillé, permettant de mettre en évidence les fautes, est la vitesse articulaire des différents axes du robot.

Pour mener à bien et évaluer l'implantation d'une maintenance prédictive, des travaux ont été effectués. Citons plus particulièrement les travaux d'évaluation des performances atteintes pour ce type de maintenance et notamment au niveau coût, [15-18], au niveau management et optimisation des programmes, [19-21]. Des comparaisons entre les différentes stratégies de maintenance, à savoir corrective, systématique, conditionnelle et prédictive ont également été menées par [22-24].

Enfin, et pour conclure cet état de l'art, citons les travaux concernant la surveillance de systèmes manufacturiers, avec une modélisation discrète des processus et une surveillance basée sur la comparaison des temps d'éxécution des tâches dans [25] et sur la gestion d'un indicateur lié au flux de production dans [26].

\section{Contexte de l'étude - Stratégie de maintenance}

Le contexte de l'étude que nous présentons a été initié par la Direction du Complexe Machinisme Agricole de Sidi Bel Abbes-Algérie où, dans un souci d'amélioration de la rentabilité, des travaux préliminaires ont mis en évidence le besoin d'optimisation de la fonction maintenance des machines d'usinage. Le cahier des charges, dans ce contexte précis, impose de fournir des lots de pièces de dimensions et de qualité de surface données. L'usinage consiste en une opération de chariotage en finition. Les contrôles qualité étant liés à la rugosité des pièces usinées, nous avons proposé de coupler cet impératif du cahier des charges à certaines actions de maintenance préventive sur l'outil de production, [27] et [28].

Dans l'approche que nous suivrons, nous préconisons, d'une part, une mise en place d'une maintenance systématique de l'outil de coupe pour pallier à l'usure normale de cet outil et nous proposons, d'autre part, une maintenance prédictive pour répondre aux fautes répertoriées que sont l'usure prématurée et le bris d'outil. Ces deux fautes ont pour conséquence une détérioration de l'état de surface de pièces mécaniques après usinage, se traduisant par une variation de la rugosité. C'est donc cette dernière, la fonction $R(t)$ où $t$ est le temps 


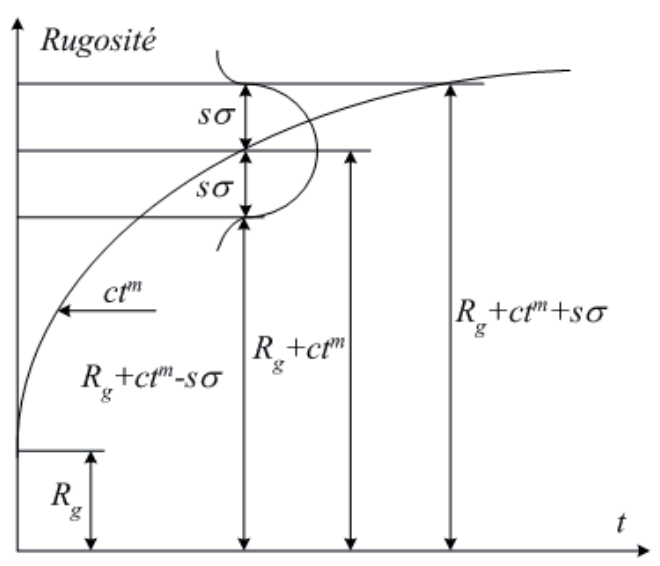

Fig. 1. Rugosité théorique.

d'usinage, que nous retenons comme indicateur central de l'étage de surveillance.

Puisque que nous ne disposons pas dans notre laboratoire de moyens de mesure en ligne de la rugosité, nous avons dû procéder à la mesure de cette rugosité de façon légèrement différée. La modélisation de la rugosité est une étape cruciale de ce travail et constitue l'objet de la section suivante.

\section{Modélisation de la rugosité}

La rugosité des surfaces des pièces usinées dépend de plusieurs facteurs. Certains sont systématiques telles les conditions géométriques de l'outil de coupe associées aux conditions cinématiques du processus de génération. Elles se modélisent par un terme constant noté rugosité de génération $R_{\mathrm{g}}$. D'autres facteurs sont associés à l'usure de l'outil et aux erreurs aléatoires d'usinage, modélisées par des fonctions déterministes ou aléatoires. Ces deux derniers facteurs s'additionnent généralement à $R_{\mathrm{g}}$.

[29] et [30] ont montré que $R(t)$ peut s'exprimer, de façon déterministe, comme une combinaison linéaire de $R_{\mathrm{g}}$ et d'un terme $c t^{m}$, où les paramètres $c$ et $m$ dépendent des conditions de coupe utilisées lors de l'usinage.

$$
R(t)=R_{\mathrm{g}}+c t^{m}
$$

Les deux paramètres $c$ et $m$ sont généralement obtenus par des techniques d'identification.

Les erreurs aléatoires d'usinage se caractérisent par une dispersion importante sur les résultats expérimentaux des mesures de $R(t)$. Il est montré en [31] que la dispersion des mesures présente une répartition d'après la loi normale (LaplaceGauss). Cette répartition est centrée, de moyenne arithmétique nulle $(\mu=0)$ et d'écart type $\sigma$.

En tenant compte de ces erreurs pour une probabilité $(P)$, et en posant $s$ la variable de répartition de la loi normale, l'équation (1) est alors bornée par un intervalle de confiance, c'est-à-dire :

$$
R_{\mathrm{g}}+c t^{m}-s \sigma \leq R(t) \leq R_{\mathrm{g}}+c t^{m}+s \sigma
$$

La variation de la rugosité est alors illustrée en figure 1 .

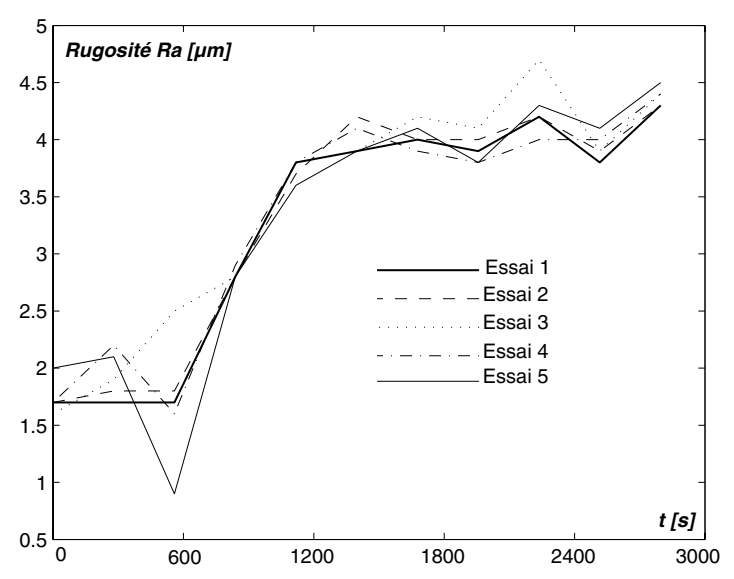

Fig. 2. Rugosité mesurée.

\section{5 Étude de cas - Identification}

Le processus d'usinage porte sur un couple outilmatière composé d'un carbure métallique de nuance P20 pour ce qui est de l'outil, et d'un acier à traitement thermique de nuance $\mathrm{C} 38$, de diamètre $100 \mathrm{~mm}$ et de longueur $150 \mathrm{~mm}$, pour ce qui est de la pièce. La rugosité maximale imposée par le dessin de définition est : $R_{\mathrm{a}}=R_{\mathrm{M}}=4,8 \mu \mathrm{m}$. Le critère $R_{\mathrm{a}}$ (rugosité moyenne arithmétique) est celui utilisé par [27], étant donné qu'il révèle les détails avec une bonne précision. Afin de satisfaire à cette contrainte de rugosité, une étude préliminaire a été menée pour déterminer les conditions optimales de coupe (vitesse de coupe, avance et géométrie de l'outil). Cette étude est détaillée dans [31] et a donné les résultats listés dans le tableau 1.

\subsection{Identification de $R_{\mathrm{g}}, c, m$, et $\sigma$}

Les mesures ont été effectuées grâce à un rugosimètre électronique à palpeur inductif (Hommel Werke), avec une longueur de base de $2 \mathrm{~mm}$ et un cut-off de $0,75 \mathrm{~mm}$. Ces mesures présentant une dispersion notable, il a été nécessaire de les réaliser sur un minimum de 5 séries de 10 pièces chacune, d'après les techniques de mesures statistiques en petite quantité [32]. Les résultats des mesures sont présentés figure 2, leur interprétation permet de décomposer $R(t)$ suivant deux intervalles distincts :

$-0<t \leq 540 \mathrm{~s}: R(t)$ est approximativement constante, sa valeur correspond à $R_{\mathrm{g}}$.

$-t>540 \mathrm{~s}: R(t)$ suit une variation de type $c t^{m}$.

L'ensemble des données relatives aux 5 courbes est traité par la technique classique des moindres carrés [31] et permet d'identifier les valeurs respectives de $c, m$, ainsi que la valeur de $\sigma$ obtenue par identification de la plus grande valeur de l'écart type des rugosités mesurées. Ces 4 paramètres sont donnés dans le tableau 2. 
Tableau 1. Conditions de coupe optimale.

\begin{tabular}{ccccccc}
\hline$V_{\mathrm{c}}\left(\mathrm{m} \cdot \mathrm{min}^{-1}\right)$ & $a\left(\mathrm{mn} \cdot \mathrm{tr}^{-1}\right)$ & $p(\mathrm{~mm})$ & $\alpha\left(^{\circ}\right)$ & $\gamma\left(^{\circ}\right)$ & $r_{\epsilon}(\mathrm{mm})$ & outil \\
\hline $220-280$ & 0,1 & 0,5 & 6 & 8 & 0,5 & $P 20$ \\
\hline
\end{tabular}

Tableau 2. Valeurs de $R_{\mathrm{g}}, c, m$ et $\sigma$.

\begin{tabular}{cccc}
\hline$R_{\mathrm{g}}$ & $c$ & $m$ & $\sigma$ \\
\hline $1,79 \mu \mathrm{m}$ & 1 & 0,102 & 0,27 \\
\hline
\end{tabular}

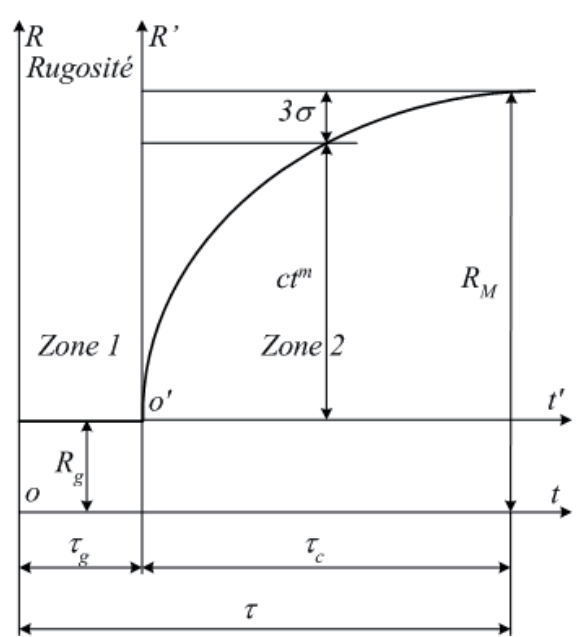

Fig. 3. Changement de base.

\section{Maintenance systématique - Usure systématique de l'outil de coupe}

\subsection{Durée de vie de l'outil de coupe}

L'usure normale de l'outil de coupe est généralement prise en charge par une maintenance systématique. Cette dernière repose sur la durée de vie de l'outil de coupe autorisant un temps de cycle d'usinage compatible avec la contrainte de rugosité imposée aux pièces. Cette détermination de la durée de vie de l'outil de coupe peut être déduite d'abaques ou grâce à des logiciels, quand ceux-ci prennent en charge le couple outil-matière utilisé, ou déterminée de façon plus précise quand le modèle de durée de vie est établi.

La valeur maximale de la rugosité $\left(R_{\mathrm{M}}\right)$ est imposée par le dessin de définition de la pièce, comme limite extrême à ne pas dépasser. La variable de répartition de la loi normale a pour valeur $s=3$ pour un intervalle de confiance à 99,7\% .

D'après les résultats expérimentaux ci-dessus, on peut considérer que la rugosité dans le premier intervalle est constante et procéder à un changement de base $\left(t o R\right.$ à $\left.t^{\prime} o^{\prime} R^{\prime}\right)$ où l'on définit les temps $\tau, \tau_{\mathrm{g}}$ et $\tau_{\mathrm{c}}$ comme indiqués sur la figure 3 .

Des relations (1) et (2), on peut écrire :

$$
R_{\mathrm{g}}+c \tau_{\mathrm{c}}^{m}+s \sigma \leq R_{\mathrm{M}}
$$

à la limite :

$$
R_{\mathrm{g}}+c \tau_{\mathrm{c}}^{m}+s \sigma=R_{\mathrm{M}}
$$

d'où

$$
\tau_{\mathrm{c}}=\left(\frac{R_{\mathrm{M}}-R_{\mathrm{g}}-s \sigma}{c}\right)^{\frac{1}{m}}
$$

qui représente le temps du cycle d'usinage relatif au second intervalle, auquel il faut additionner $\tau_{\mathrm{g}}$ pour obtenir $\tau$, temps maximum du cycle d'usinage satisfaisant à la contrainte de rugosité :

$$
\tau=\tau_{\mathrm{g}}+\tau_{\mathrm{c}}
$$

Nous déduisons directement de la figure $2: \tau_{\mathrm{g}} \approx 540 \mathrm{~s}$. Les équations (5) associées aux valeurs du tableau 2 et (6) permettent de déterminer $\tau \approx 2815 \mathrm{~s}$, soit :

$$
\tau \approx 47 \min
$$

La maintenance systématique de l'outil consistera donc, à $\tau \approx 47 \mathrm{~min}$ en un changement de cet outil ou en son réaffutage.

\section{Maintenance prédictive de l'outil de coupe}

La maintenance systématique ne prend en compte que l'usure systématique de l'outil. Mais nous savons que toute usure prématurée de l'outil de coupe se traduira obligatoirement par une augmentation du rebut des pièces. Inversement, le changement d'outil n'est en rien justifié et devient donc prématuré si l'outil de coupe présente une longévité plus grande.

Nous considérons dans ce travail 2 types de fautes concernant l'outil de coupe, d'une part, son usure prématurée et d'autre part, le bris de son bec. Notons :

- $f_{1}$ : faute d'usure prématurée de l'outil de coupe

- $f_{2}$ : faute de bris du bec de l'outil de coupe (microéclat).

\subsection{Détection et localisation des fautes - Principe}

Compte tenu du fait que nous disposons du modèle analytique de $R(t)$, l'approche de détection des fautes basée sur l'utilisation du modèle est tout à fait indiquée. Le principe de cette méthode est de comparer les sorties mesurées du système $y_{\text {mesuré }}(t)$ et les mêmes sorties estimées $y_{\text {estimé}}(t)$ grâce au modèle, comme indiqué en figure 4. La même entrée $u(t)$ est appliquée au système et à son modèle. En l'absence de faute, ces deux sorties sont identiques (au bruit et aux erreurs de modélisation près) et le signal $r(t)$, appelé résidu, tend vers zéro. Toute occurrence de faute se traduit par une déviation de la sortie mesurée et $r(t)$ est différent de zéro. Une logique de décision, pouvant 


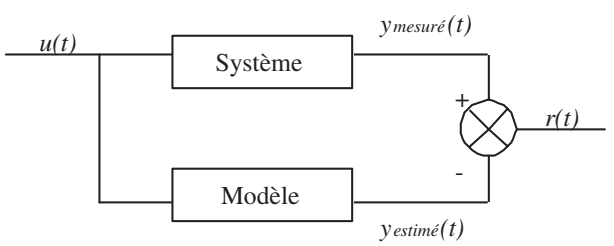

Fig. 4. Construction du résidu.

se réduire à un seuillage, permet de décider de l'occurrence de la faute. Le seuil est choisi afin de prendre en compte le niveau du bruit et les incertitudes de modélisation.

Cette approche est souvent utilisée pour la surveillance des systèmes continus. La détection se fait en ligne et en temps réel, le modèle est programmé sur calculateur et fonctionne en parallèle avec le système.

Notre approche est une variante de la procédure décrite en figure 4. Les valeurs du tableau 1 $\left(V_{\mathrm{c}}, a, f, \alpha, \gamma\right.$ et $\left.r_{\epsilon}\right)$ sont les entrées appliquées au tour (système réel) tandis que celles du tableau $2\left(R_{\mathrm{g}}, c, m\right.$ et $\left.\sigma\right)$ sont les entrées du calculateur (modèle).

La sortie mesurée correspond à la rugosité $R_{\mathrm{a}}(t)$ et la sortie estimée $R(t)$ est obtenue par (1). Le résidu $r(t)$ s'obtient alors par :

$$
r(t)=R_{\mathrm{a}}(t)-R(t)
$$

Notons $S_{\mathrm{H}}$ et $S_{\mathrm{B}}$ respectivement les seuils haut et bas, déterminés expérimentalement et permettant de prendre en compte le bruit et les erreurs de modélisation. Nous utilisons le niveau de confiance extrême relatif à la loi normale $(99,7 \%)$ qui permet de poser $S_{\mathrm{B}}=-3 \sigma$ et $S_{\mathrm{H}}=+3 \sigma$.

- si : $r(t) \in\left[S_{\mathrm{B}}, S_{\mathrm{H}}\right] \rightarrow$ mode sans faute,

- si : $r(t) \notin\left[S_{\mathrm{B}}, S_{\mathrm{H}}\right] \rightarrow$ occurrence d'une faute.

La localisation de la faute s'effectuera par l'analyse du résidu et notamment du taux de dépassement du seuil. Les expérimentations font apparaître que l'évolution du résidu, au moment de l'occurrence de la faute, est caractéristique de cette dernière, ceci, il est vrai, du fait du nombre limité des fautes prises en compte.

\subsection{Expérimentation et résultats}

Cette section traite de la validation des algorithmes de détection-localisation établis ci-dessus. Pour ce faire, nous avons effectué 3 types d'essais. Le premier, noté essai 0 , s'est déroulé sans injection de faute, tandis que les fautes $f_{1}$ et $f_{2}$ ont été introduites dans les 2 essais suivants. Une seule faute a été injectée par essai.

Sur toutes les figures relatives à l'évolution de $r(t)$, 2 traits horizontaux en pointillé représentent les 2 valeurs $S_{\mathrm{B}}=-3 \sigma=-0,81$ et $S_{\mathrm{H}}=+3 \sigma=0,81$. La rugosité maximale $R_{\mathrm{M}}=4,8 \mu \mathrm{m}$ est également représentée par un trait horizontal en pointillé sur les figures exprimant $R_{\mathrm{a}}(t)$.

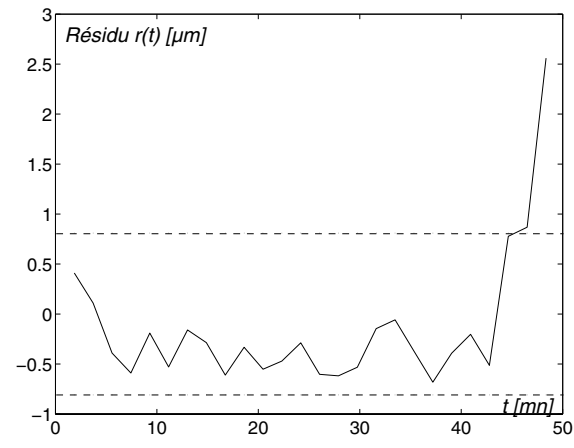

Fig. 5. Résidu $r(t)$ en mode sans faute.

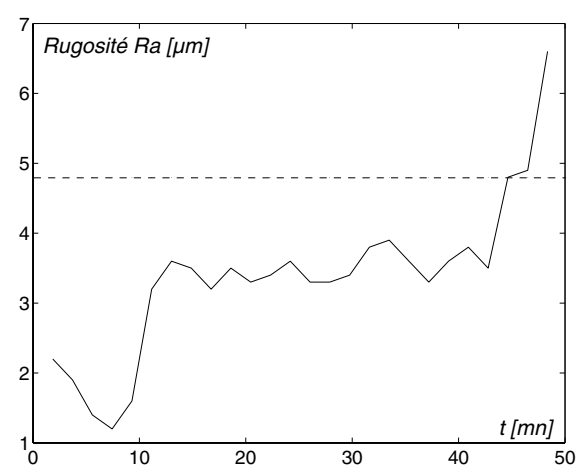

Fig. 6. Rugosité $R_{\mathrm{a}}$ en mode sans faute.

\subsubsection{Essai 0 - Mode sans faute}

L'essai 0 a été mené de façon à amener l'outil en fin de vie afin de pouvoir comparer ce temps avec le temps calculé en (6). Cette procédure a nécessité l'usinage de 26 pièces.

Nous notons d'après la figure 5 que durant $\tau_{\mathrm{g}}$, défini en figure 3, la rugosité de génération n'est pas constante mais présente une décroissance due probablement à la formation préalable de l'arrondi du bec de l'outil de coupe. Au bout de $t \approx 45 \mathrm{~min}, r(t)$ prend une valeur significative car la durée de vie maximale de l'outil est atteinte et on a $R_{\mathrm{a}}>R_{\mathrm{M}}$, voir figure 6 . Il est à noter que ce temps $t \approx 45$ min est proche de $\tau \approx 47$ min. Ce dernier, d'après (7), est le temps de référence pour la procédure liée à la maintenance systématique.

\subsubsection{Essai 1 - Injection de $f_{1}$ : faute d'usure prématurée de l'outil}

Les usures prématurées des outils sont généralement dues à la mauvaise qualité de ces derniers ou à de mauvaises caractéristiques du matériau des pièces. Même si ces fautes sont relativement rares, elles n'en restent pas moins lourdes de conséquences car tout un lot de pièces peut à ce moment être non conforme.

Nous avons simulé cette faute en utilisant, en lieu et place de l'outil neuf, un outil usagé.

$\grave{\mathrm{A}} t \approx 5 \min r(t)>S_{\mathrm{H}}$, comme indiqué figure 7 , une faute est détectée. Cependant, la rugosité affiche une valeur $R_{\mathrm{a}}(t)<R_{\mathrm{M}}$ et la maintenance prédictive 


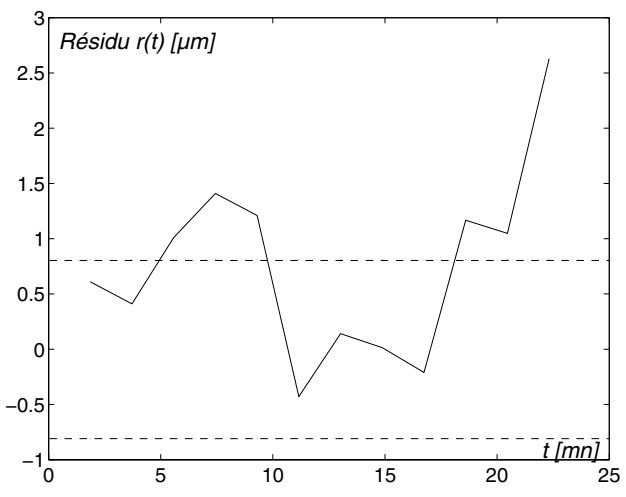

Fig. 7. Résidu $r(t)$ avec faute $f_{1}$.

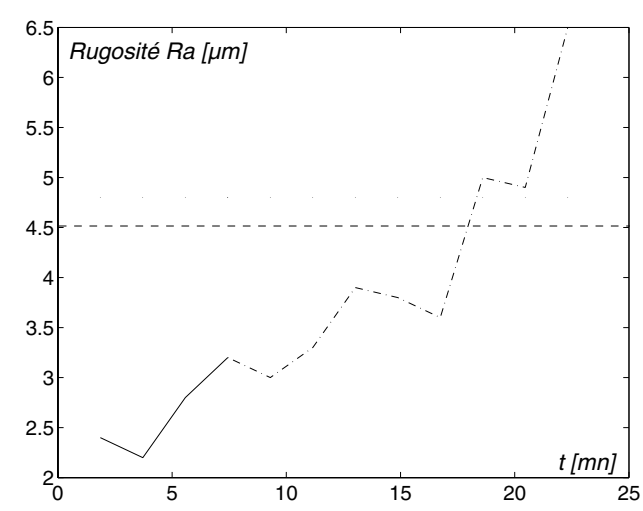

Fig. 8. Rugosité $R_{\text {a }}$ avec faute $f_{1}$.

prend ici tout son sens. Il faut alors estimer le temps pour lequel l'usinage de pièces est encore possible avec une rugosité inférieure à $R_{\mathrm{M}}$. Cette étape est en soi un problème non trivial, et si elle devait être automatisée, elle nécessiterait des développements conséquents qui constituent une étape future de nos travaux.

Toutefois dans le cas présent, afin de fixer les idées, une approche de résolution est de réaliser la prédiction, à partir de l'instant de détection de la faute, en utilisant la fenêtre temporelle [5 min, 7,5 min], voir figure 8. Du fait de l'allure de la courbe jusqu'à cet instant précis, une approximation de la courbe par une fonction affine est satisfaisante. L'instant d'intervention, obtenu par intersection de la fonction affine avec le seuil haut, est alors $t \approx 16 \mathrm{~min}$.

Notons qu'indépendamment de cette procédure de prédiction du temps, l'usinage a été mené à son terme afin de constater l'évolution de $R_{\mathrm{a}}(t)$. Comme indiqué figure 8, courbe en trait mixte, cette fonction peut être considérée comme affine par morceaux. Elle présente des changements de pente mais reste globalement croissante. Le temps limite pour lequel $R_{\mathrm{a}}(t)>R_{\mathrm{M}}$ est $t \approx 18 \mathrm{~min}$. La prédiction émise s'avère donc satisfaisante puisque l'on constate une différence de 2 min, correspondant à un temps approximatif d'usinage de 2 pièces.

\subsubsection{Essai 2 - Injection de $f_{2}$ : faute de bris de l'outil}

Un endommagement du bec de l'outil est effectué en $t \approx 16 \mathrm{~min}$. Il en résulte une variation rapide

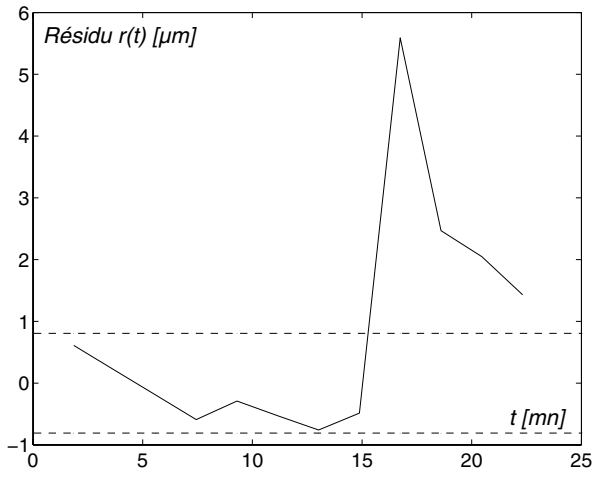

Fig. 9. Résidu $r(t)$ avec faute $f_{2}$.

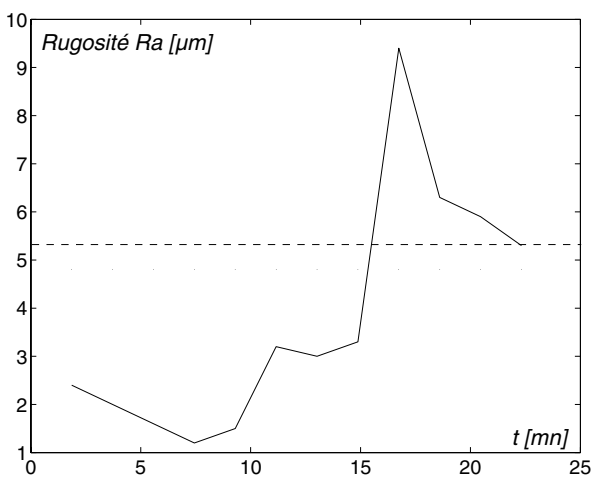

Fig. 10. Rugosité $R_{\mathrm{a}}$ avec faute $f_{2}$.

de $r(t)$ du fait de la variation elle même rapide de $R_{\mathrm{a}}(t)$, comme indiqué figures 9 et 10 . À ce moment et contrairement à $f_{1}$, il n'y a pas matière à prédiction, la rugosité $R_{\mathrm{a}}(t)$ est instantanément supérieure à $R_{\mathrm{M}}$ et la seule décision possible est l'arrêt d'urgence.

Il est donc essentiel que l'effet de la faute se traduise par une détérioration lente de la rugosité afin de disposer d'une plage de temps de prédiction significative.

\section{Conclusion}

Le travail que nous présentons dans cet article a pour but de montrer les avantages liés à la mise en place d'une maintenance prédictive, notamment en usinage. Cette étude permet de valider complètement le principe de détection des fautes considérées, même si des ajustements s'avèreront sûrement nécessaires lorsque le système de mesure de la rugosité en ligne sera adopté. Le choix de la rugosité, comme indicateur significatif de l'état de l'outil de coupe, et donc comme élément central de la procédure d'implantation de la maintenance prédictive, est d'autant plus judicieux qu'il constitue le principal paramètre qualité à valider lors des contrôles finaux. Cette considération peut faciliter la prise de décision concernant l'investissement d'un système de mesure de la rugosité en ligne.

Nous avons montré que la maintenance prédictive permet la prise en compte de fautes telle l'usure prématurée de l'outil ou le bris d'outil, et cet état de fait constitue le principal avantage par rapport à 
la maintenance systématique qui ne prend en charge que l'usure systématique de l'outil. Le temps de vie de l'outil, calculé grâce au modèle et utilisé en maintenance systématique, s'est avéré assez proche des temps trouvés lors des expérimentations en mode sans faute.

\section{Références}

[1] J.C. Laprie, Guide de la sûreté de fonctionnement, Cépaduès, 1995

[2] R. Isermann, Model-based fault-detection and diagnosis-status and applications, Ann. Rev. Control 29 (2005) 71-85

[3] M. Bartys, R. Patton, M. Syfert, S. Heras, J. Quevedo, Introduction to the damadics actuator fdi benchmark study, Control Eng. Practice 14 (2006) $577-596$

[4] F. Noureddine, Fault tolerance in robots, in AVCS'04, IFAC Genova, 2004

[5] H.B Jun, D. Kiritsis, M. Gambera, P. Xirouchakis, Predictive algorithm to determine the suitable time to change automotive engine oil, Computer Ind. Eng. Elsevier 51 (2006) 671-683

[6] C. Fu, L. Ye, Y. Liu, R. Yu, B. Iung, Y. Cheng, Y. Zeng, Predictive maintenance in intelligentcontrol-maintenance-management system for hydroelectric generating unit, IEEE Transactions on Energy Conversion 19 (2004) 179-186

[7] M.C. Garcia, M.A. Sanz-Bobi, J. Del Pico, Simap: System for predictive maintenance-application to the health condition monitoring of a windturbine gearbox, Computers Ind. 57 (2006) 552-568

[8] M.C. Pan, H.V. Brussel, P. Sas, Intelligent joint fault diagnosis of industrial robots, Mech. Syst. Signal Proc. 12 (1998) 571-588

[9] E. Gilabert, A. Arnaiz, Intelligent automation systems for predictive maintenance: A case study, Robotics and Computer-integrated Manufacturing 22 (2006) 543-549

[10] D. Bansal, D.J. Evans, B. Jones, Application of a real-time predictive maintenance system to a production machine system, Int. J. Machine Tools Manufacture 45 (2005) 1210-1221

[11] D. Bansal, D.J. Evans, B. Jones, Bjest: A reverse algorithm for the real-time predictive maintenance system, Int. J. Machine Tools Manufacture 46 (2006) 1068-1078

[12] N. Orhan, S. Aktürk, V. Celik, Vibration monitoring for defect of rolling element bearings as a predictive maintenance tool: Comprehensive case studies, NDTE Int. 39 (2006) 293-298

[13] S. Velarde-Suarez, R. Ballesteros-Tajadura, J.P. Hurtado-Cruz, A predictive maintenance procedure using pressure and acceleration signals from a centrifugal fan, Appl. Acoustic 67 (2006) 49-61

[14] F. Noureddine, Conception d'un outil logiciel graphique pour l'analyse comportementale d'un robot en mode dégradé, Revue Internationale de CFAO et d'Informatique Graphique 11 (1996) 199-214

[15] R.J. Murry, B.F. Mitchell, Cost saving from a practical predictive maintenance program, in Annual Reliability and Maintainability Symposium, 1994, pp. 206-209
[16] M. Crowder, J. Lawless, On a scheme for predictive maintenance, Eur. J. Operational Res. 176 (2007) 1713-1722

[17] M.C. Eti, S.O.T. Ogaji, S.D. Probert, Reducing the cost of preventive maintenance (pm) through adopting a proactive reliability-focused culture, Applied Energy 83 (2006) 1235-1248

[18] L.M. Maillart, S.M. Pollock, Cost-optimal conditionmonitoring for predictive maintenance of 2-phase systems, IEEE Trans. on Reliability 51 (2002) 322-330

[19] A. Grall, L. Dieulle, C. Bérenguer, M. Roussignol, Continous-time predictive-maintenance scheduling for a deteriorating system, IEEE Trans. Reliability 51 (2002) 141-150

[20] M.C. Carnero, Selection of diagnostic techniques and instrumentation in a predictive maintenance program, a case study, Decision Support System 91 (2003) 945-963

[21] M.C. Carnero, An evaluation system of the setting up of predictive maintenance programmes, Reliability Engineering and System Safety, 2006

[22] L.R. Contreras, C. Modi, A. Pennathur, Integrating simulation modeling and equipment condition diagnostics for predictive maintenance strategies, A case study, in Winter Simulation Conference (2002) pp. $1289-1294$

[23] L. Lu, J. Jiang, Analysis of on-line maintenance strategies for k-out-of-n standby safety systems, Reliability Engineering and System Safety 92 (2007) 144-155

[24] X. Zhou, L. Xi, J. Lee, Reliability-centered predictive maintenance scheduling for a continuously monitored system subject to degradation, Reliability Engineering and System Safety 92 (2007) 530-534

[25] Z.S. Abazi, Z. Bouredji, Monitoring and predictive maintenance : Modeling and analyse of fault latency, Comp. Ind. 57 (2006) 504-515

[26] F. Ly, A.K.A. Toguyeni, E. Craye, Indirect predictive monitoring in flexible manufacturing systems, Robotics and Computer-integrated Manufacturing 16 (2000) 321-338

[27] D.A. Axinte, R.C. Dewes, Surface integrity of hot work tool steel after high speed milling-experimental data and empirical models, Materials Proc. Tech. 127 (2002) 325-335

[28] S.A. Coker, Y.C. Shin, In-process control of surface roughness with tool wear via ultrasonic sensing, in American Control Conference Washington, 1996

[29] A.M. Alam, R. Chieragatti, M. Akamatsu, Y. Caumel, Effect of machining procedures on the surface state of a low carbon steel, Scr. Mat. 37 (1997) 1559-1567

[30] R. Noureddine, A. Benamar, F. Noureddine, Intégration d'une technique de maintenance prédictive de l'outil de coupe dans une cellule d'usinage robotisée, in PENTOM 2005, Marrakech, 2005

[31] A. Benamar, G. Inglebert, M. Boumahrat, Durée de vie d'un carbure p20 pour le chariotage d'un acier c38 à rugosité contrôlée, Mécanique \& Industries 6 (2005) 635-640

[32] M. Bouchetara, A. Guermat, K. Belaib, Étude du phénomène d'usure des pièces des pompes d'injection par la méthode taguchi, Mécanique \& Industries 4 (2003) 221-228 